\title{
Massive hemorrhage secondary to metastatic testicular carcinoma
}

\author{
LEWIS N. CUNNINGHAM, DO \\ PHILLIP GINSBERG, DO \\ SAMUEL MANFREY, DO \\ LEONARD H. FINKELSTEIN, DO, FACOS
}

Massive bleeding secondary to metastatic foci of testicular carcinoma is a rare finding. Two cases of metastatic testicular carcinoma in which massive intraabdominal and gastrointestinal hemorrhage occurred are reported. The first patient, who had metastasis to the duodenum and stomach, first underwent surgery because of the concern that chemotherapy might result in rapid tumor necrosis and bowel perforation. The bleeding was controlled, postoperative chemotherapy was administered, and the patient was alive 15 months after chemotherapy. In the second case, in which metastasis was to the liver and lungs, aggressive chemotherapy was begun because of the patient's poor pulmonary status. Three days later, the patient began to hemorrhage. Operative and massive resuscitative measures failed, and the patient died shortly after surgery.

Severe bleeding from metastatic carcinoma is uncommon, but when it does occur, it usually is in patients who have metastatic lesions of the testicle, prostate gland, stomach, or breast. ${ }^{1,2}$ Bleeding is presumed to be caused by erosion of the tumor mass into adjacent, pre-existing blood vessels. It is also possible that blood vessels produced by neovascularization may be a potential source of hemorrhage.

Serious complications for metastatic cancer of testicular origin are rare. Watkins, ${ }^{1}$ Lock, ${ }^{2}$ and Saliba and associates ${ }^{3}$ each reported one case of gastrointestinal hemorrhage secondary to metastatic testicular carcinoma.

We add two more cases to the literature. In one patient, massive intrahepatic and intra-abdominal hemorrhage was thought to have resulted from rapid tumor shrinkage and vascular erosion. Gastrointestinal bleeding was controlled in the other patient.

\section{Report of cases \\ Case 1}

A 25-year-old man with a right testicular mass, which had progressed in size and nodulation over a two-month period, was first seen in the emergency room of a local hospital. He complained of right-sided testicular pain of three weeks' duration, intermittent nausea and emesis, and a 4-lb weight loss.

Physical examination revealed the abdomen to be soft, and no abnormal mass or organomegaly was palpated. Right costovertebral angle tenderness was elicited. A slightly tender, rock-hard mass was palpated on the upper pole of the right testicle; the contralateral testicle was normal. The patient was admitted for further examination.

Chest roentgenography and tomography of the chest had negative findings. Scrotal ultrasonography revealed a $2.7 \times 2.5 \times 2.2 \mathrm{~cm}$, solid testicular mass on the right. Computed tomography (CT) demonstrated an intra-aortacaval node measuring $2.5 \times 2.5 \times 2.0 \mathrm{~cm}$, which possibly was indicative of metastatic disease. The $\beta$-human chorionic gonadotropin and $\alpha$-fetoprotein levels were in the normal range. Two days after admission, rightsided inguinal exploration, testicular biopsy, and radical orchiectomy were performed. A pathologic diagnosis of teratocarcinoma (teratoma and embryonal carcinoma) was made. The margins were free of tumor. Postoperative $\alpha$-fetoprotein and $\beta$-human chorionic gonadotropin levels remained normal. Repeat CT showed no changes. The patient was discharged but scheduled for retroperitoneal lymph node dissection for staging purposes.

Two weeks after surgery, the patient was seen by us for a second opinion regarding retroperitoneal lymph node dissection, which we recommended. However, the patient refused treatment.

Two months later, complaining of severe back and abdominal pain of 12 hours' duration, he was readmitted to the hospital. Physical examination revealed generalized abdominal guarding, but no rebound tenderness. There was continued concern that metastatic disease was present. The pain was controlled, and the patient was discharged three days later with instructions to undergo radical retroperitoneal lymphadenectomy.

$\mathrm{He}$ again refused, and he was lost to follow-up until seven months after his initial presentation, when he was readmitted with complaints of weakness and vertigo. The hemoglobin value was $5.8 \mathrm{~g} / \mathrm{dL}$, and a stool specimen was positive for occult blood. The $\beta$-human chorionic gona- 


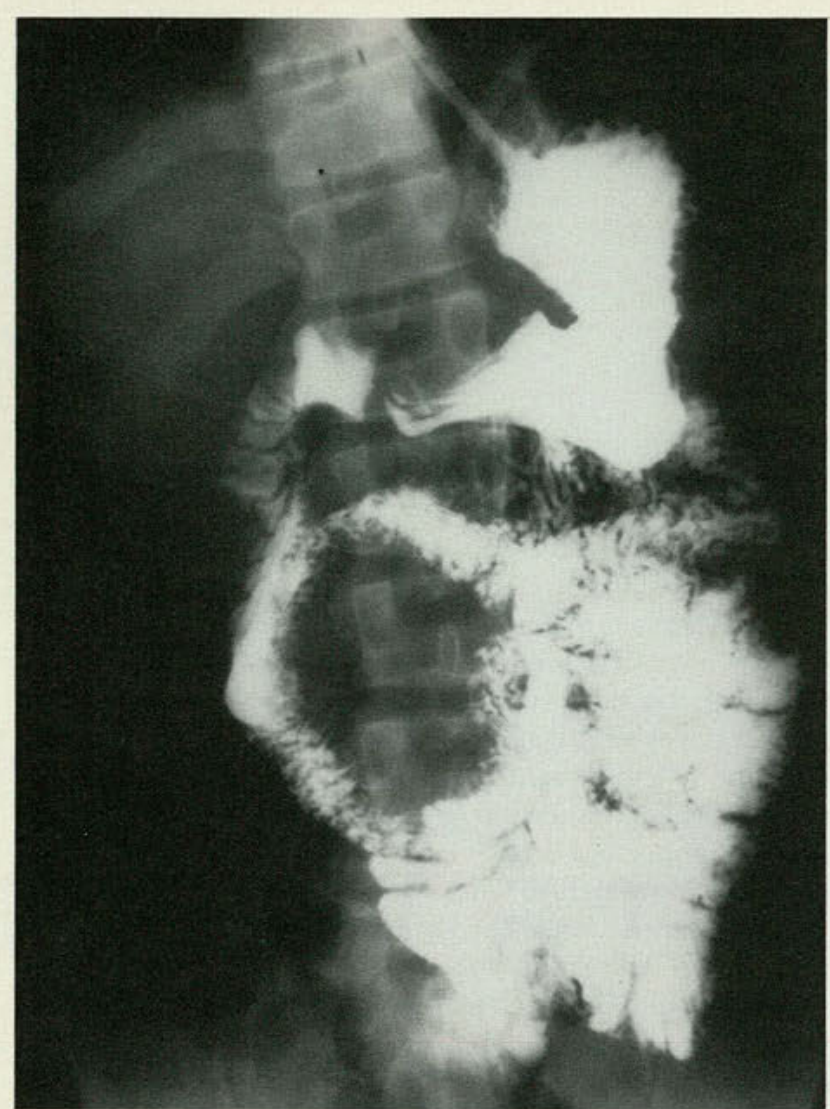

Fig 1. Space-occupying lesion of the upper abdomen (case 1).

dotropin level was $515 \mathrm{mU} / \mathrm{mL}$, and the liver enzyme values were elevated. Multiple transfusions were required to achieve hemodynamic stability. An upper gastrointestinal radiographic series revealed an $8-\mathrm{cm}$ retroperitoneal tumor displacing the stomach and duodenum (Fig 1). Esophagogastroduodenoscopy demonstrated esophagitis, bile reflux, and antritis; duodenal ulcer was not visualized. The second portion of the duodenum had an irregular, friable intraluminal mass (Fig 2).

Biopsy specimens were obtained, and the frozen sections were positive for metastatic embryonal cell carcinoma. Consultants from the hematology and oncology department thought that aggressive chemotherapy would result in rapid tumor necrosis and shrinkage, which, in turn, would cause bowel perforation.

Therefore, the patient was taken to the operating room for resection of the invading tumor and involved bowel and for restoration of bowel continuity. Metastatic disease was noted to involve the second and third portions of the retroperitoneum. There was no metastatic disease in the liver or spleen. The second, third, and fourth portions of the duodenum, as well as a major portion of the stomach, were excised. Gastrojejunostomy and Roux-en$\mathrm{Y}$ anastomosis were performed along with the tubal gastrotomy and jejunostomy.

The pathology report indicated the presence of metastatic embryonic cell carcinoma of the duodenum and jejunum. The postoperative recovery period was unevent-

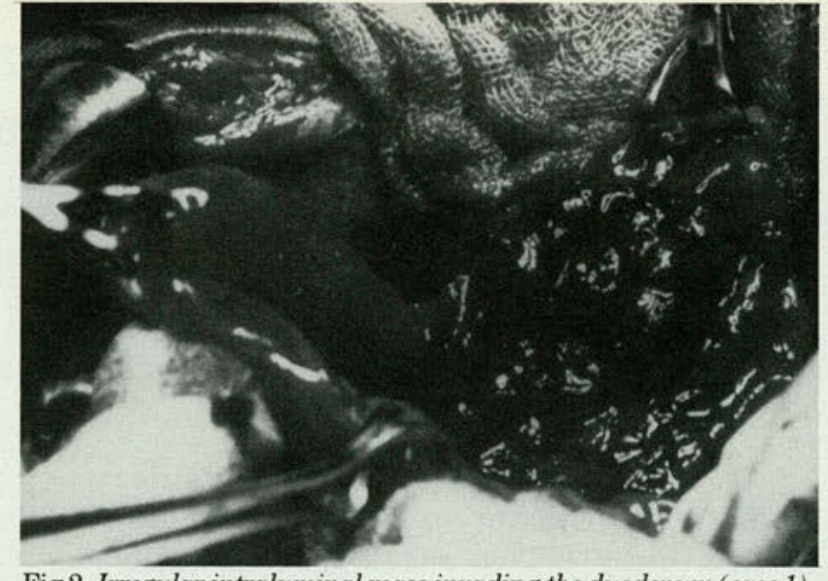

Fig 2. Irregular intraluminal mass invading the duodenum (case 1).

ful, and the patient was scheduled for aggressive chemotherapy. He received four cycles of chemotherapy, including bleomycin, VP-16, and cisplatin. When he was to receive his fifth cycle of chemotherapy, he refused.

The patient's $\beta$-human chorionic gonadotropin and $\alpha$-fetoprotein levels returned to normal ranges during chemotherapy, and he was known to be alive and well 15 months later.

\section{Case 2}

An 18-year-old man with a painless right-sided testicular mass, which had been present for eight to ten months, was admitted. The patient reported a 20 - to 25 -lb weight loss during that time.

Physical examination revealed a right testicle that was three to four times larger than the contralateral side. It was roughly $6 \mathrm{~cm}$ in diameter, hard to palpation, and highly indicative of malignancy.

The patient was cachectic and dehydrated. The hemoglobin level was $7.8 \mathrm{~g} / \mathrm{dL}$. The $\beta$-human chorionic gonadotropin value was $200,000 \mathrm{mU} / \mathrm{mL}$, and the liver enzyme and bilirubin values were elevated.

Testicular ultrasonography was positive for a large hyperechoic testicular mass with multiple cystic areas in the right testis (Fig 3). These findings were compatible with testicular neoplasm. CT showed the lungs to have multiple rounded areas of high density in the posterior lung parenchyma, as well as a density measuring $10 \times 14 \mathrm{~cm}$ adjacent to the vertebral column on the right side (Fig 4).

Two days after admission and transfusion, the patient underwent right-sided orchiectomy, and testicular teratocarcinoma was diagnosed. It was decided to initiate chemotherapy immediately because of the patient's poor pulmonary status. A regimen of cyclophosphamide, vinblastine sulfate, actinomycin $\mathrm{D}$, and bleomycin was followed with cisplatin therapy.

Five days postoperatively (three days after initiation of chemotherapy), the patient complained of abdominal pain. Abdominal examination revealed guarding, rigidity, and rebound tenderness. The hemoglobin level was $8.9 \mathrm{~g} / \mathrm{dL}$, and the platelet count was $52,000 / \mathrm{cu} \mathrm{mm}$. The 
chest film showed early onset of adult respiratory distress syndrome in addition to metastatic disease.

The patient was taken to the operating room, and exploratory laparotomy revealed massive intra-abdominal hemorrhage from necrotic hepatic metastasis. Partial resection of the left lobe of the liver and enucleation of the right hepatic mass (Fig 5) were performed. Active hemorrhage was noted from every cut surface; this finding was compatible with disseminated intravascular coagulation. The patient required massive volume resuscitation (39 units of packed red blood cells, six units of platelets, and seven units of fresh frozen plasma).

In the intensive care unit, the patient's hemoglobin level was $5.5 \mathrm{~g} / \mathrm{dL}$. After massive crystalloid and colloid resuscitation, the patient continued to have exsanguination, and he died shortly thereafter.

\section{Discussion}

Massive intra-abdominal and gastrointestinal hemorrhage caused by testicular metastasis is rare. Cases of solitary and multiple metastasis to the stomach, duodenum, and jejunum that resulted in massive upper gastrointestinal bleeding have been reported. ${ }^{1-3}$

Patients with testicular tumors, especially choriocarcinoma and teratocarcinoma, frequently present with the effects of their metastasis. The primary lesion may be quite small, but with hematogenous and lymphatic spread, there may be widespread metastasis in the lungs, liver, lymph nodes, and mediastinum. In a report by Dixon and Moore ${ }^{4}$ on seven cases of metastatic testicular carcinoma discovered at autopsy, metastatic distribution was as follows: periaortic and iliac nodes, $100 \%$; each lung, $100 \%$; liver, $86 \%$; intestine, $71 \%$; and spleen, adrenal glands, and brain, $56 \%$.

Ballanger ${ }^{5}$ reported a case of testicular metastasis to the stomach that presented with symptoms of ulcer disease. The patient lived 12 years after resection. In Prosser's ${ }^{6}$ study of 358 cases of testicular cancer, $20 \%$ of the patients had metastasis on presentation. The most frequently involved lymph nodes were the paraortic nodes, followed by the iliac, supraclavicular, and inguinal nodes.

It is uncommon to find serious complications of gastrointestinal metastasis of testicular origin. Johnson and Strauss ${ }^{7}$ studied eight cases of intussusception and found one that resulted from metastatic testicular carcinoma.

Saliba and associates ${ }^{3}$ reported a case of choriocarcinoma of the testicle with metastasis to the jejunum, in which the patient presented with gastrointestinal hemorrhage requiring resection. In the case of choriocarcinoma of the testicle reported by Lock, ${ }^{2}$ severe gastrointestinal bleeding from metastatic sites to the jejunum and stomach re-

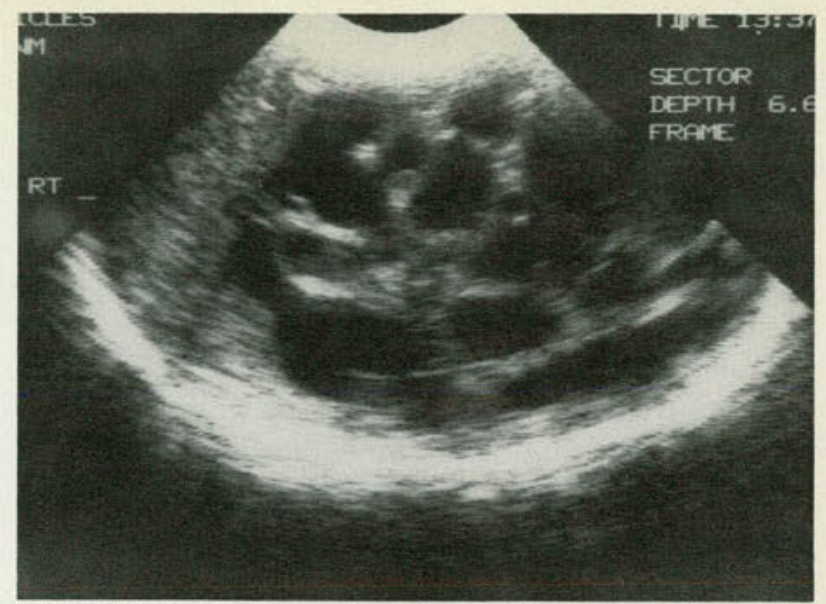

Fig 3. Multicystic, hyperechoic mass of the right testis (case 2).

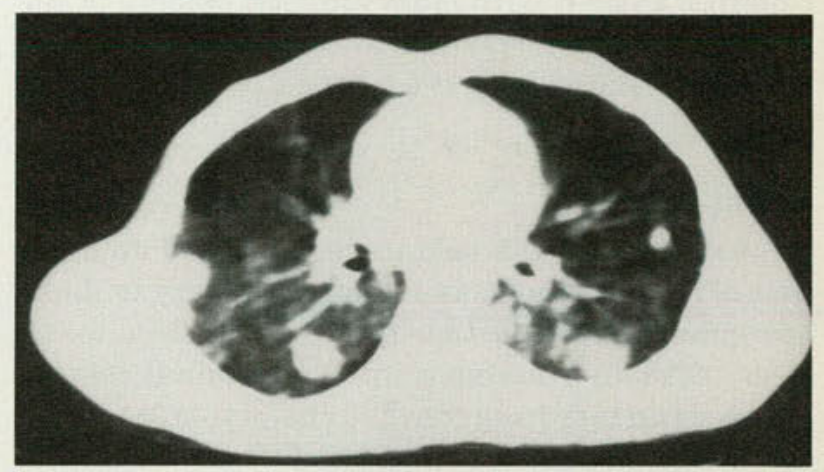

Fig 4. Testicular carcinoma metastasis to the lung (case 2).

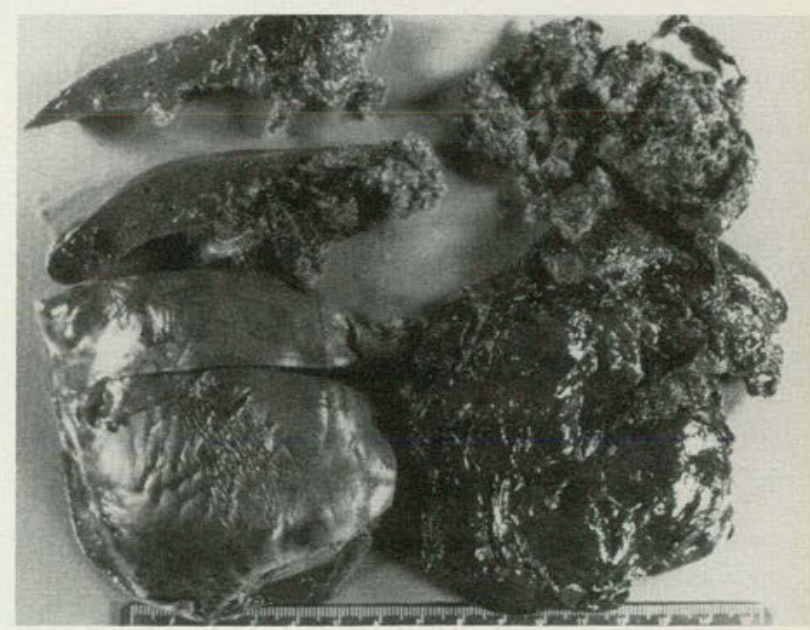

Fig 5. Resected right hepatic mass (case 2).

quired extensive surgical resection. Watkins ${ }^{1}$ reported a case of intra-abdominal hemorrhage secondary to vascular disruption from metastatic seminoma.

In our first case, massive gastrointestinal hemorrhage resulted from metastasis to the duodenum 
and stomach, which was caused by direct extension of involved lymphatic metastasis. Surgery was mandated by the patient's condition and our concern that aggressive chemotherapy might result in bowel perforation. Partial gastrectomy, gastrojejunostomy, and Roux-en-Y anastomosis were performed, and the patient was discharged and scheduled for chemotherapy.

Our second case is unusual in that it involves presumed choriocarcinoma metastasizing from the testicle to the liver, but no choriocarcinoma was found in the resected testicle. Hemorrhage occurred from necrosis of the metastatic liver mass, which was caused by aggressive chemotherapy. Rapid tumor lysis was reported by Doty and associates ${ }^{8}$ to result in bronchial perforation and abscess formation in a patient with embryonic carcinoma of the testicle that metastasized to the lung. This rapid necrosis resulted from the tumor's response to chemotherapy.

\section{Conclusions}

In our review of the literature, we found no other case of rupture and hemorrhage secondary to choriocarcinoma of the testicle that metastasized to the liver. Although massive intra-abdominal hemorrhage secondary to aggressive chemotherapy is uncommon, one must be aware of this unusual complication with testicular tumors, which may be very responsive to the chemotherapeutic agents in current use.

In case number 1 , the use of chemotherapy prior to diversion of gastric contents could have resulted in duodenal perforation following rapid shrinkage of the metastatic tumor.

1. Watkins GL: Massive hemoperitoneum resulting from rupture of a seminoma in an undescended testicle. J Urol 1970;103:447-448.

2. Lock MR: Testicular tumor presenting as hematemesis. Postgrad Med J 1975;602:857-859.

3. Saliba NS, Sawyer KC, Hall WW, et al: Choriocarcinoma in the male patient presenting as gastrointestinal hemorrhage. Am J Surg 1966;112:764-769.

4. Dixon FJ, Moore RA: Tumors of male sex organs, in Atlas of Tumor Pathology. Washington, DC, United States Armed Forces Institute of Pathology, 1952.

5. Ballanger R: Gastric metastasis of a testicular carcinoma operated on twelve years earlier. J Urol Nephrol 1963;69:531-532.

6. Prosser TM: Tumors of the testis. J Royal Coll Edin 1985;9:85-106.

7. Johnson WA, Strauss FH: Intussusception of the small intestine produced by metastatic carcinoma. Surgery 1952;32:991-996.

8. Doty JD, Lynch GR, Smith FE, et al: Bronchial rupture as a complication of response to combination chemotherapy in metastatic testicular carcinoma. $J$ Urol 1984;132:135-136.

From the Division of Urology, Department of Surgery, Hospital of the Philadelphia College of Osteopathic Medicine, Philadelphia.

Reprint requests to Dr Finkelstein, GSB Bldg, Suite 714, Belmont and City Ave, Bala-Cynwyd, PA 19004. 



\section{CONVENIENT B.I.D. DOSAGE Dosage guidelines TABLETS (ciprofloxacin $\mathrm{HCl} /$ Miles) Mild/Moderate Infections: $500 \mathrm{mg}$ q12h Severe/Complicated Infections: 750 mg q12h}

\author{
CIPRO $^{*}$ \\ (ciprofloxacin hydrochloride/Miles) \\ TABLETS \\ BRIEF SUMMARY \\ CONSULT PACKAGE INSERT FOR FULL PRESCRIBING INFORMATION \\ INDICATIONS AND USAGE
}

Cipro is indicated for the treatment of infections caused by susceptible strains of the designated micro-

organisms in the conditions listed below.
Lower Respiratory Infections caused by Escherichia coli, Klebsiella pneumoniae, Enterobacter cloacae. Proteus mirabilis, Pseudomonas aeruginosa. Haemophilus influenzae, Haemophilus parainfluenzae, and Streptococcus pneumoniae.

Skin and Skin Structure Infections caused by Escherichia coli, Klebsiella pneumoniae, Enterobacter cloacae Proteus mirabilis, Proteus vulgaris, Providencia stuartii, Morganella morganii, Citrobacter freundii, Pseudomonas aeruginosa. Staphylococcus aureus (penicillinase and nonpenicillinase-producing strains) Staphylococcus epidermidis, and Streptococcus pyogenes.

Bone and Joint Infections caused by Enterobacter cloacae, Serratia marcescens, and Pseudomonas aeruginosa.

Urinary Tract Infections caused by Escherichia coli, Klebsiella pneumoniae, Enterobacter cloacae, Serratia marcescens, Proteus mirabilis, Providencia rettgeri, Morganella morganii, Citrobacter diversus, Citrobacte freundii, Pseudomonas aeruginosa, Staphylococcus epidermidis, and Streptococcus faecalis.

Infectious Diarrhea caused by Escherichia coli (enterotoxigenic strainsl, Campylobacter jejuni, Shigella flexneri, and Shigella sonner" when antibacterial therapy is indicated

*Efficacy for this organism in this organ system was studied in fewer than 10 infections.

Appropriate culture and susceptibility tests should be performed before treatment in order to isolate and identify organisms causing infection and to determine their susceptibility to ciprofloxacin. Therapy with Cipro* may be initiated before results of these tests are known; once results become available appropriate therapy should be continued. As with other drugs, some strains of Pseudomonas aeruginosa may develop resistance fairly rapidly during treatment with ciprofloxacin. Culture and susceptibility testing performed periodically during therapy will provide information not only on the therapeutic effect of the antimicrobial agent but also on the possible emergence of bacterial resistance. CONTRAINDICATIONS

A history of hypersensitivity to ciprofloxacin is a contraindication to its use. A history of hypersensitivity to other quinolones may also contraindicate the use of ciprofloxacin.

CIPROFLOXACIN SHOULD NOT BE USED IN CHILDREN, ADOLESCENTS, OR PREGNANT WOMEN. The oral administration of ciprofloxacin caused lameness in immature dogs. Histopathological examination of the weightbearing joints of these dogs revealed permanent lesions of the cartilage. Related drugs such as nalidixic acid cinoxacin, and norfloxacin also produced erosions of cartilage of weight-bearing joints and other signs of
arthropathy in immature animals of various species (SEE ANIMAL PHARMACOLOGY SECTION IN FULL arthropathy in immature animas
PRESCRIBING INFORMATION)

\section{PRECAUTIONS}

General: As with other quinolones, ciprofloxacin may cause central nervous system (CNS) stimulation, which may lead to tremot, restlessness, lightheadedness, confusion, and very rarely to hallucinations or convulsive seizures. Therefore, ciprofloxacin should be used with caution in patients with known or suspected CNS
disorders, such as severe cerebral arteriosclerosis or epilepsy. or other factors which predispose to seizures (SEE ADVERSE REACTIONSI

Quinolones may also cause anaphylactic reactions and cardiovascular collapse. Anaphylactic reactions may require epinephrine and other emergency measures

Crystals of ciprofloxacin have been observed rarely in the urine of human subjects but more frequently in the urine of laboratory animals. Crystalluria related to ciprofloxacin has been reported only rarely in man, because human urine is usually acidic. Patients receiving ciprofloxacin should be well hydrated, and alkalinity of the urine should be avoided. The recommended daily dose should not be exceeded. Alteration of the dosage regimen is necessary for patients with impairment of renal function (SEE DOSAGE AND ADMINISTRATION SECTION IN FULL PRESCRIBING INFORMATION)

Drug Interactions: Concurrent administration of ciprofloxacin with theophylline may lead to elevated plasma concentrations of theophylline and prolongation of its elimination half-life. This may result in increased risk of theophylline-related adverse reactions. If concomitant use cannot be avoided, plasma levels of theophylline should be monitored and dosage adjustments made as appropriate.

Antacids containing magnesium hydroxide of aluminum hydroxide may interfere with the absorption of ciprofloxacin, resulting in serum and urine levels lower than desired; concurrent administration of these agents with ciprofloxacin should be avoided.

Probenecid interferes with the renal tubular secretion of ciprofloxacin and produces an increase in the level of ciprofloxacin in the serum. This should be considered if patients are receiving both drugs concomitantly. As with other broad-spectrum antibiotics, prolonged use of ciprofloxacin may result in overgrowth of nonsusceptible organisms. Repeated evaluation of the patient's condition and microbial susceptibility testing is essential. If superinfection occurs during therapy appropriate measures should be taken

Information for Patients: Patients should be advised that ciprofloxacin may be taken with or without meals The preferred time of dosing is two hours after a meal. Patients should also be advised to drink fluids liberally and not take antacids containing magnesium of aluminum concomitantly of within two hours after dosing Ciprofloxacin may cause diziness or lightheadedness: therefore patients should know how they react to this drug before they operate an automobile or machinery or engage in activities requiring mental alertness of coordination.

Carcinogenesis, Mutagenesis, Impairment of Fertility: Eight in vitro mutagenicity tests have been

conducted with ciprofloxacin and the test results are listed below

Salmonella/Microsome Test (Negative)

E. coli DNA Repair Assay (Negative)

Mouse Lymphoma Cell Forward Mutation Assay (Positive)

Chinese Hamster $\mathrm{V}_{29}$ Cell HGPRT Test (Negative)

Syrian Hamster Embryo Cell Transformation Assay (Negative)

Saccharomyces cerevisiae Point Mutation Assay (Negative)

Saccharomyces cerevisiae Mitotic Crossover and Gene Conversion Assay (Negative)

Rat Hepatocyte DNA Repair Assay (Positive)

Thus, two of the eight tests were positive, but the following three in vivo test systems gave negative results: Rat Hepatocyte DNA Repair Assay

Micronucleus Test (Mice)

Dominant Lethal Test (Mice)

Long-term carcinogenicity studies in animals have not yet been completed.

to six times the usual daily human dose and have revealed no evidence of impaired fertility or harm to the fetus due to ciprofloxacin. In rabbits, as with most antimicrobial agents, ciprofloxacin $(30$ and $100 \mathrm{mg} / \mathrm{kg}$ orally) produced gastrointestinal disturbances resulting in maternal weight loss and an increased incidence of abortion.
No teratogenicity was observed at either dose. After intravenous administration, at doses up to $20 \mathrm{mg} / \mathrm{kg}$, maternal toxicity was produced, and no embryotoxicity or teratogenicity was observed. There are, however, adequate and well-controlled studies in pregnant women. SINCE CIPROFLXACIN, LIKE ORER DRUS IN CLEE WARNINGS] Nursing Mothers: It is not known whether ciprofloxacin is excreted in human milk; however, it is known th ciprofloxacin is excreted in the milk of lactating rats and that other drugs of this class are excreted in human m Because of this, and because of the potential for serious adverse reactions from ciprofloxacin in nursing infants. decision should be made to discontinue nursing or to discontinue the drug, taking into account the importance the drug to the mother

Pediatric Use: Ciprofloxacin should not be used in children because it causes arthropathy in immature anima (SEE WARNINGS)

\section{ADVERSE REACTIONS}

Ciprofloxacin is generally well tolerated. During clinical investigation, 2.799 patients received 2.868 courses the drug. Adverse events that were considered likely to be drug related occurred in $7.3 \%$ of courses, possit related in $9.2 \%$, and remotely related in $3.0 \%$. Ciprofloxacin was discontinued because of an adverse event $3.5 \%$ of courses, primarily involving the gastrointestinal system $(1.5 \% \mathrm{l}$, skin $10.6 \%$, and central nervous syst

$0.4 \%)$
The most frequently reported events, drug related or not, were nausea $(5.2 \%)$ diarrhea $(2.3 \%)$ vomiting $12.0 \%$ abdominal pain/discomfort $1.7 \%$ ! headache $(1.2 \%)$ restlessness $(1.1 \%)$ and rash $(1.1 \%)$

Additional events that occurred in less than $1 \%$ of ciprofloxacin courses are listed below. Those typical quinolones are italicized

GASTROINTESTINAL: (See above), painful oral mucosa, oral candidiasis, dysphagia, intestinal perforatio gastrointestinal bleeding.

CENTRAL NERIVUS SYSTEM: (See above), dizziness, lightheadedness, insomnia, nightmares, hallucin tions, manic reaction, irritability, tremor, ataxia, convulsive seizures, lethargy, drowsiness, weaknes malaise, anorexia, phobia, depersonalization, depression, paresthesia.

SKIN/HYPERSENSITIVITY: (See above), pruritus, urticaria, photosensitivity, flushing, fever, chil angioedema, edema of the face, neck, lips, conjunctivae or hand's, cutaneous candidiasis, hyperpigment tion, erythema nodosum.

Allergic reactions ranging from urticaria to anaphylactic reactions have been reported

SPECIAL SENSES: blurred vision, disturbed vision, Ichange in color perception, overbrightness of ligh decreased visual acuity, diplopia, eye pain, tinnitus, bad taste.

MUSCULOSKELETAL: joint or back pain, joint stiffness, achiness, neck or chest pain, flare-up of gout. RENAL/UROGENITAL: interstitial nephritis, renal failure, polyuria, urinary retention, urethral bleedin vaginitis, acidosis.

CARDIONASCULAR: palpitations, atrial flutter, ventricular ectopy, syncope, hypertension, angina pector myocardial infarction, cardiopulmonary arrest, cerebral thrombosis.

RESPIRATORY: epistaxis, laryngeal or pulmonary edema, hiccough, hemoptysis, dyspnea, bronchospas pulmonary embolism.

Most of these events were described as only mild or moderate in severity, abated soon after the drug w discontinued, and required no treatment.

In several instances, nausea, vomiting, tremot, restlessness, agitation, or palpitations were judged nvestigators to be related to elevated plasma levels of theophylline possibly as a result of a drug interaction w ciprofloxacin

Adverse Laboratory Changes: Changes in laboratory parameters listed as adverse events without regard drug relationship

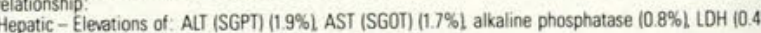

serum bilinubin $(0.3 \%)$
Hematologic - eosinophilia $(0.6 \%)$ leukopenia $(0.4 \%)$ decreased blood platelets $10.1 \%)$, elevated blo platelets $(0.1 \%)$ pancyopenia $(0.1 \%)$.

Renal - Elevations of: Serum creatinine $1.1 \%$. BUN $10.9 \%$

CRYSTALLURIA, CYLINDRURIA, AND HEMATURIA HAVE BEEN REPORTED

Other changes occurring in less than $0.1 \%$ of courses were: Elevation of serum gammaglutamyl transfera elevation of serum amylase, reduction in blood glucose, elevated uric acid, decrease in hemoglobin, aner bleeding diathesis, increase in blood monocytes, and leukocytosis
OVERDOSAGE

Information on overdosage in humans is not available. In the event of acute overdosage, the stomach should emptied by inducing vomiting or by gastric lavage. The patient should be carefully observed and given suppor treatment. Adequate hydration must be maintained. In the event of serious toxic reactions from overdos hemodialysis or peritoneal dialysis may aid in the removal of ciprofloxacin from the body. particularly if re function is compromised

\section{DOSAGE AND ADMINISTRATION}

The usual adult dosage for patients with urinary tract infections is $250 \mathrm{mg}$ every 12 hours. for patients w complicated infections caused by organisms not highly susceptible, $500 \mathrm{mg}$ may be administered every 12 hou Respiratory tract infections, skin and skin structure infections, and bone and joint infections may be trea with $500 \mathrm{mg}$ every 12 hours. For more severe or complicated infections, a dosage of $750 \mathrm{mg}$ may be given ever hours.

The recommended dosage for infectious diarrhea is $500 \mathrm{mg}$ every 12 hours.

In patients with renal impairment, some modification of dosage is recommended ISEE DOSAGE A ADMINISTRATION SECTION IN FULL PRESCRIBING INFORMATION
HOW SUPPLIED

Cipro* (ciprofloxacin HCI/Miles) is available as tablets of $250 \mathrm{mg}, 500 \mathrm{mg}$, and $750 \mathrm{mg}$ in bottles of 50 , an Unit-Dose packages of 100 (SEE FULL PRESCRIBING INFORMATION FOR COMPLETE INFORMATION)

*Due to susceptible strains of indicated pathogens. See indicated organisms in Prescribing Information.

For further information, contad the Miles Information Service: 1-800-642-4776. In VA. call collect: 703-391-7888.

\section{COMMITTED TO THERAPEUTIC EFFICIENC}

\section{M MILES}

c) October 1988 , Miles Inc.
Miles Inc.

Pharmaceutical Divisic 400 Morgan Lane West Haven, CT 06516

Printed in U.S.A. C09618 MLR-3 